Ate Visser, Roelof Stuurman, and Marc F.P. Bierkens (2004), Real time forecasting of water table depth and soil moisture profiles, in Proceedings of the $2^{\text {nd }}$ international CAHMDA workshop on: The Terrestrial Water Cycle: Modelling and Data Assimilation Across Catchment Scales, edited by A.J. Teuling, H. Leijnse, P.A. Troch, J. Sheffield and E.F. Wood, pp. 117-121, Princeton, NJ, October 25-27 


\title{
Real time forecasting of water table depth and soil moisture profiles
}

\author{
Ate Visser $^{1}$, Roelof Stuurman ${ }^{1}$, and Marc F.P. Bierkens ${ }^{1,2}$ \\ ${ }^{1}$ TNO-Institute of Applied Geoscience, Utrecht, The Netherlands \\ ${ }^{2}$ Department of Physical Geography, Utrecht University, Utrecht, The Netherlands
}

\begin{abstract}
A method is presented for online forecasting of water table depth and soil moisture profiles. The method combines a simple form of data assimilation with a moving window calibration of a deterministic model describing flow in the unsaturated zone as well as regional drainage. Previous work with physically-deterministic models (Knotters and Bierkens, 2000; Knotters and De Gooijer, 1999) has focussed on offline calibration and validation. However, availability of online measurements makes online forecasting possible. Online forecasting can be used for both drought and flood warnings, enabling more timely and accurate operational water management. Also, online calibration provides substantially better forecasts than offline calibration.
\end{abstract}

Materials and methods The groundwater data were obtained from an observation well located on the main meteorological field of the Royal Netherlands Meteorological Office at De Bilt. The field lies at the edge of an ice-pushed ridge that is a remnant from the glaciers that covered the north of the Netherlands during the Saalien ice age. Because of its proximity, the ridge is expected to be a source of regional groundwater fluxes, influencing the phreatic surface (Bierkens et al., 1999).

Besides a long series (15 years) of daily groundwater measurements, online measurements of water table depth and soil moisture (at 25, 50, 75 and $100 \mathrm{~cm}$ below surface) were available for a period of three months. Each day these measurements were sent to a central server by short message service (sms) over the consumer gsm network. From there, the data was retrieved over the internet, together with historical weather data and weather forecasts (for which the Royal Netherlands Meteorological Institute is acknowledged) to be used for calibration and forecasting.

The Soil Water Atmosphere Plant model (SWAP) (van Dam, 1997) was used, which applies the Richards equation in combination with the Mualem-Van Genuchten soil hydraulic parameters, to describe the flow of water in the unsaturated zone. A linear relationship between drainage flux and phreatic surface depth was assumed, parameterised by the drainage level of the nearest draining agent, $h_{s}$, and the drainage and infiltration resistance, $\gamma$. The bottom flux, $q_{b}$, was assumed to be constant in time here, although it might have a seasonal fluctuating component. A mobile fraction, $f_{m}$, was used to compensate for preferential flow of percolating water. In addition, to allow for a fast responding system, the saturated conductivity $K_{s}$, was calibrated. This left five parameters $\left(h_{s}, \gamma, q_{b}, f_{m}\right.$ and $\left.K_{s}\right)$ to be estimated on long series of groundwater data. The additional use of soil water content measurements also allowed for calibration of the Van Genuchten parameters $\alpha$ and $n$, strongly improving soil water profile predictions. To account for seasonal fluctuations and compensate for model error, a 31-day moving window calibration of the drainage level, $h_{s}$, was used, because this parameter is directly related to the absolute value of the water table depth.

For short lead-time forecasting with a dynamic model (here a maximum lead time of 5 days was used), two criteria are important: (1) adequate parameters and (2) a close approximation of the initial conditions. Using predictions from a 31-day moving window calibration often resulted in a large error when compared to the last available measurement, introducing an error in the initial condition for the forecast. To eliminate this error, the objective function $(O)$ for the moving 


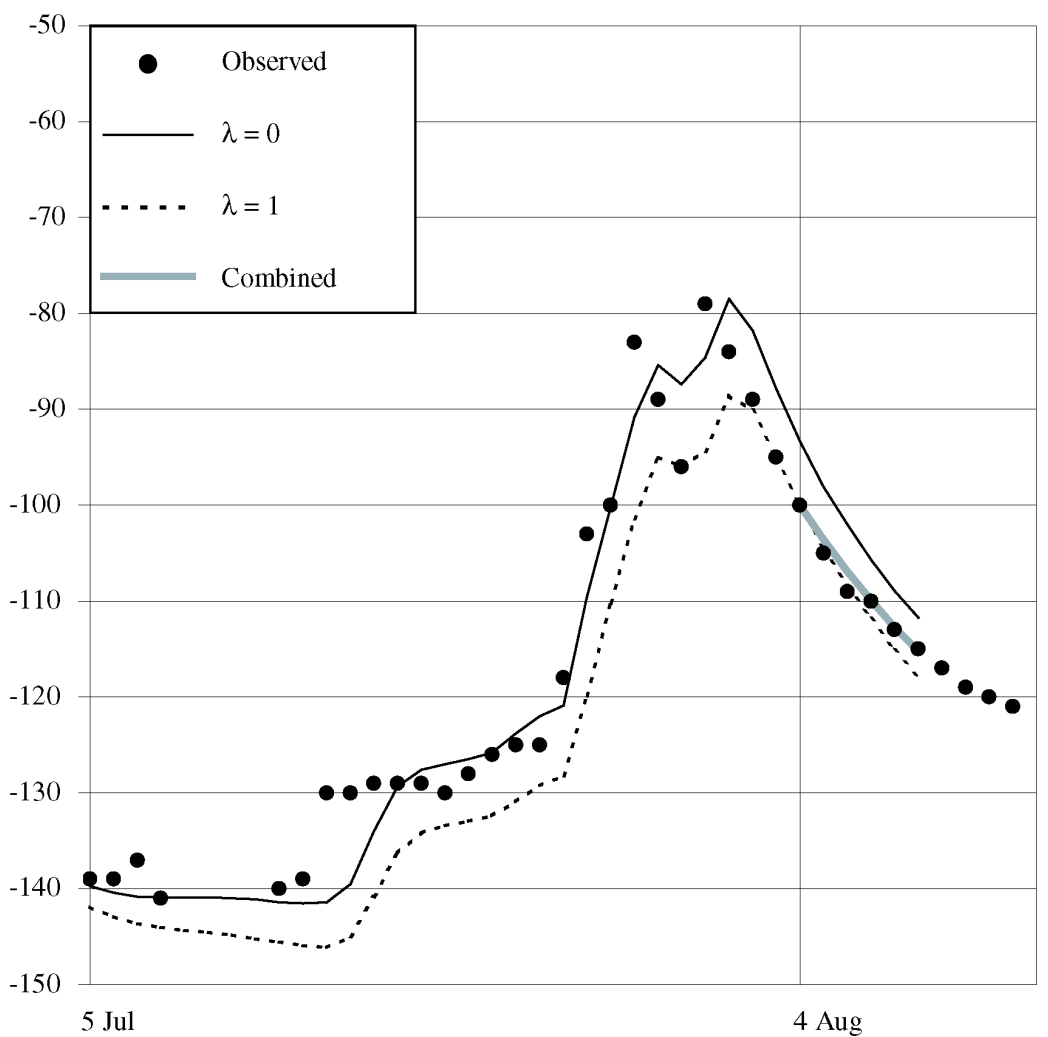

Figure 3.11: Illustration of the advantage of the combined method. Benefiting from the initial state from the $\lambda=1$ calibration and the parameter value of the $\lambda=0$ calibration, the combined method starts of well and stays in line with the observed values.

window calibration was changed and the last measurement of the calibration period was given extra weight.

$$
O=(1-\lambda) \cdot \sum_{i=1}^{n} R(i)+\lambda \cdot R(n)
$$

with $R(i)$ being the residual to the $i$-th measurement. This will force the calibration method to "work towards" the last measurement. We define $\lambda$ as the measure for the extra weight (i.e., $\lambda=0$ assigns equal weight to all measurements and $\lambda=1$ assigns only weight to the last measurement). Note that this produces a form of model adaptation that is in between calibration and a form of data assimilation, similar to Newtonian Nudging (Houser et al., 1998). Assigning more weight to the last measurement (high $\lambda$ ) will reduce the initial error, but introduces a larger parameter error. Therefore we also combined the initial condition of $\lambda=1$ and the estimated parameter of $\lambda=0$, to further improve the forecast.

The forecast method was tested on a 15-year series of historical groundwater data, as well as on simulated series for a sensitivity analysis. Finally, the combined method was applied to a short period for which both groundwater and soil moisture content measurements were available.

Results We found an optimum $\lambda$ of 0.25 when considering the overall RMSE of the forecast period (5-day lead time). Larger values of $\lambda$ resulted in large errors in the model parameter, whereas smaller values of $\lambda$ result in large errors in initial values. This is shown in Figure 3.11 where the prediction error is plotted against lead-time for several values for $\lambda$ as well as the combined 


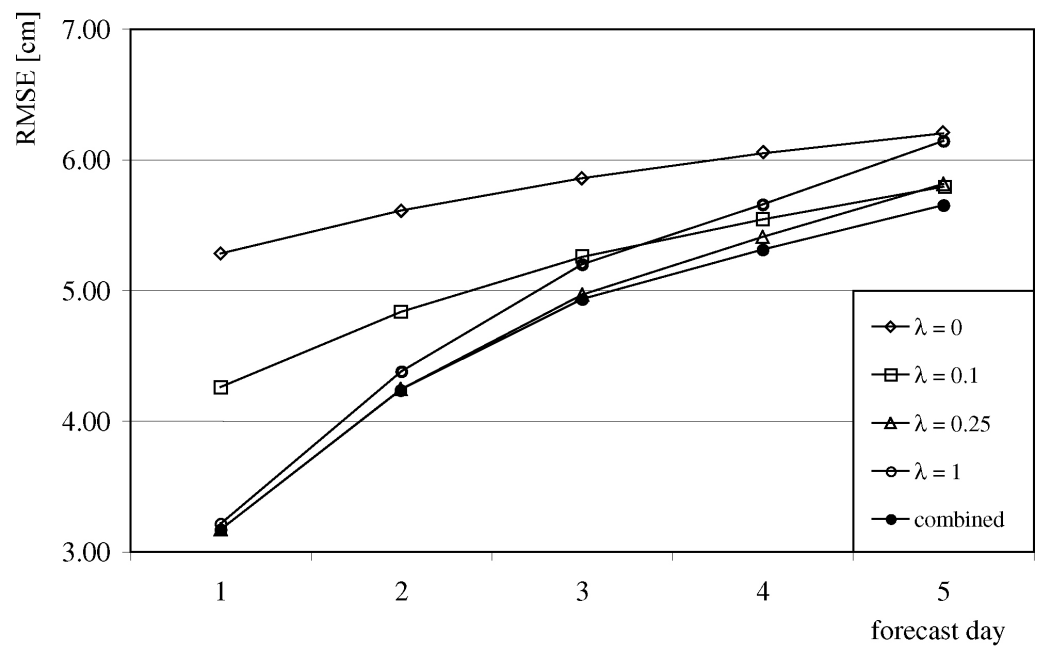

Figure 3.12: Evolution of prediction error over forecast period with several values for weighing $\lambda$ and combined method.

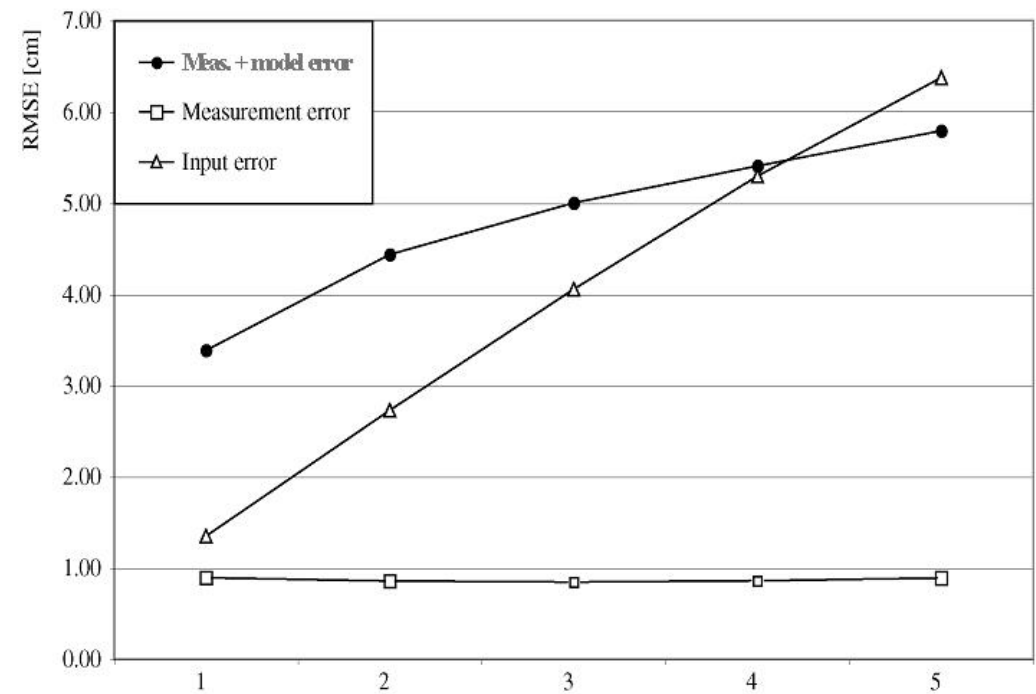

Figure 3.13: Evolution of the prediction error of the water table depth over the forecast period for three sources of error, model error in real data, and measurement error and input error from simulated time series. 

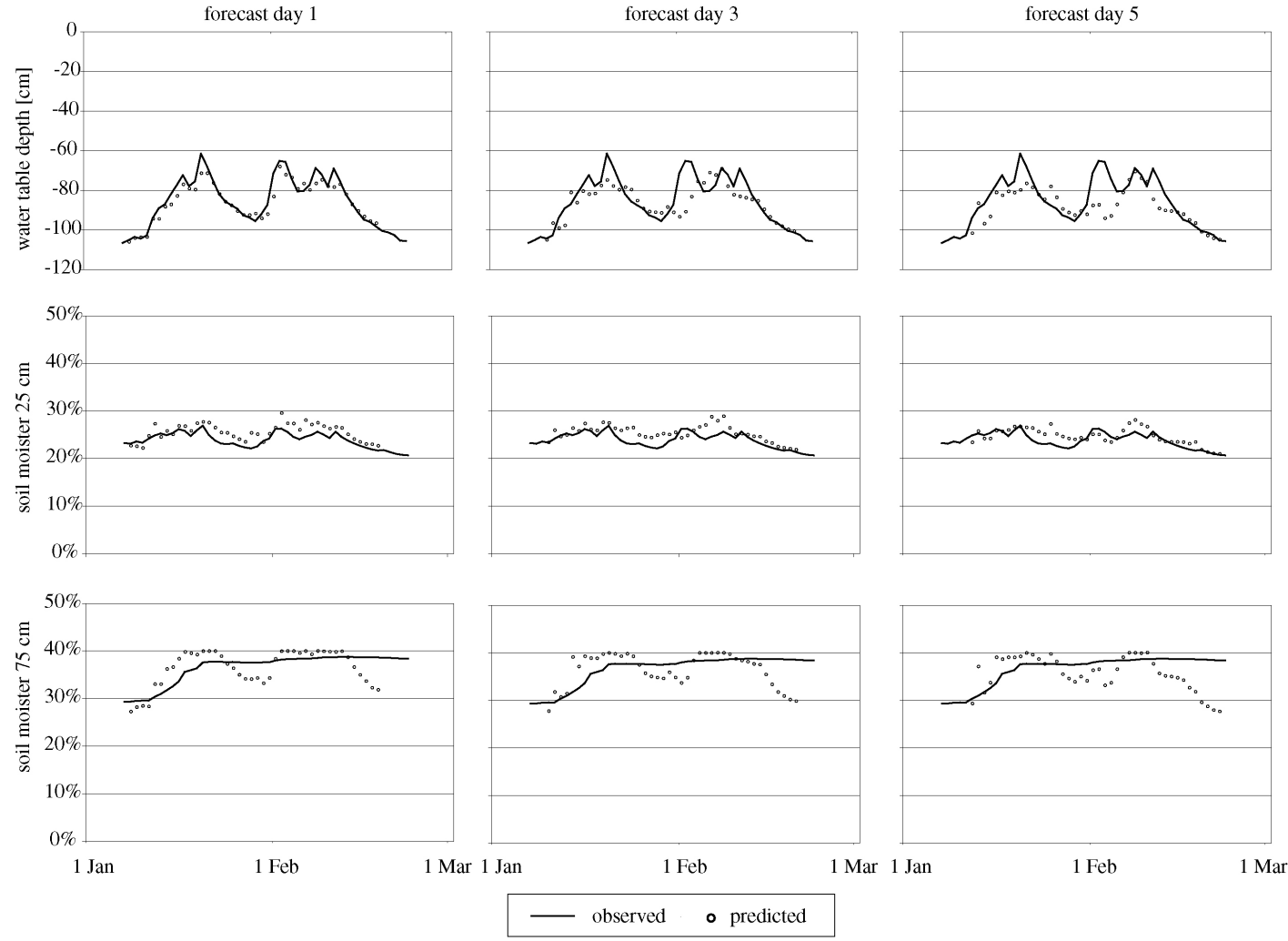

Figure 3.14: Time series of observed and predicted water table depths and soil moisture content of the $1^{\text {st }}, 3^{\text {rd }}$ and $5^{\text {th }}$ forecast day.

method. The combination of $\lambda=0$ and $\lambda=1$ calibration benefits from both a very small initial error and a better parameter value, and gives the best overall forecast in terms of RMSE, better than using a $\lambda$ of 0.25 . This effect is further illustrated in Figure 3.12.

Three types of error can be distinguished: model error, measurement error and input error (errors in the weather forecast). The difference between historical groundwater data and forecasts using observed meteorological data provided an estimate of the combined model error and measurement error. Noise added to simulated time series (mimicking measurement error) or input variables (mimicking errors in weather forecast) gave insight into the other two sources of error. As shown by Figure 3.13, the model error is largest, up to the $4^{\text {th }}$ forecast day, when the error from the weather forecast becomes as large.

Finally we applied the whole scheme for online forecasts of ground water level and soil moisture profiles (5 day maximum lead time), using weather forecasts and the combined moving window calibration of both groundwater and soil moisture data. Figure 3.14 shows some time series of observed and forecasted water table depth and soil moisture content. Differences in error statistics of soil water content hereby reflect differences in dynamics; see Figure 3.15 . The $25 \mathrm{~cm}$ soil water content series remains the same in error during the forecast period, reflecting diffusion of soil moisture mitigating the error from the weather forecast. The $50 \mathrm{~cm}$ soil water content shows similar behaviour, whereas the $75 \mathrm{~cm}$ soil water content is more influenced by ground water level fluctuations and therefore follows the pattern of groundwater error statistics, deteriorating over the forecast period.

Conclusions We introduced a method for on-line forecasting of water table depth and soil moisture profiles. The method is based on a combination of a moving window calibration of the determinis- 

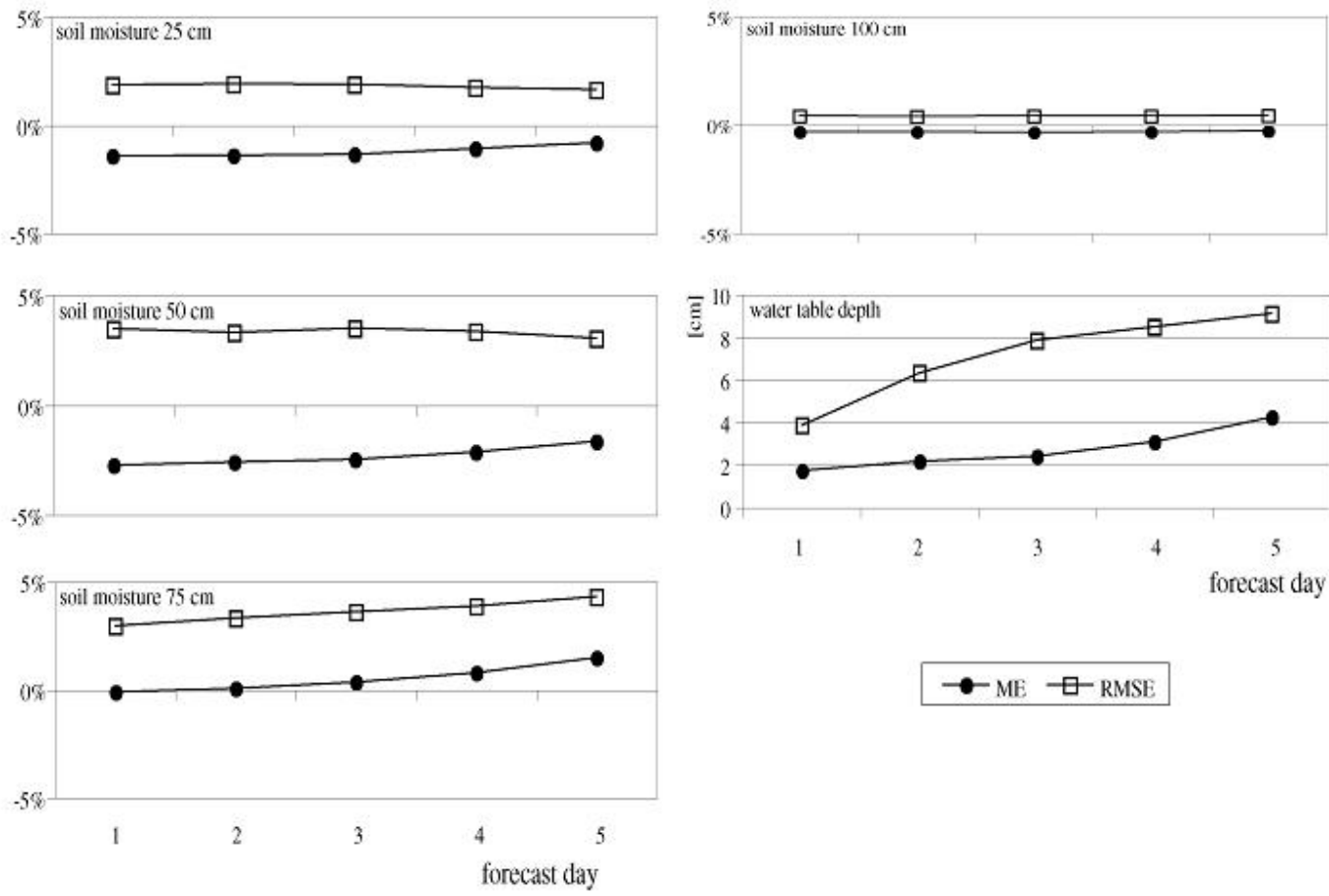

Figure 3.15: Evolution of prediction error over forecast period of groundwater level and soil moisture profile.

tic model of the unsaturated zone SWAP and a simple form of data assimilation. Moving window calibration improves the forecast compared to an offline model. Adding a simple form of data assimilation improved the forecast further.

The model error is the largest source of error, except for lead times larger than 4 days, when the error resulting from the error in weather forecast becomes as large. Forecasts of groundwater level and soil moisture content below the topsoil deteriorate over the forecast period. Diffusion processes in the topsoil mitigate the deteriorating prediction forecast, resulting in a constant accuracy of the predicted water content over the forecast period. 


\section{Bibliography}

Bierkens, M., M. Knotters, and F. Van Geer, Calibration of transfer function-noise models to sparsely or irregularly observed time series, Water Resour. Res., 35, 1741-1750, 1999.

Houser, P., W. Shuttleworth, J. Famiglietti, H. Gupta, K. Syed, and D. Goodrich, Integration of soil moisture remote sensing and hydrologic modeling using data assimilation, Water Resour. Res., 34(12), 3405-3420, 1998.

Knotters, M., and M. Bierkens, Physical basis of time series models for water table depths, Water Resour. Res., 36, 181-188, 2000.

Knotters, M., and J. De Gooijer, TARSO modeling of water table depths, Water Resour. Res., 35, 695-705, 1999.

van Dam, J., Theory of SWAP version 2.0: simulation of water flow, solute transport and plant growth in the Soil-Water-Atmosphere-Plant environment, Tech. rep., DLO Winand Staring Centre, Wageningen, 1997. 